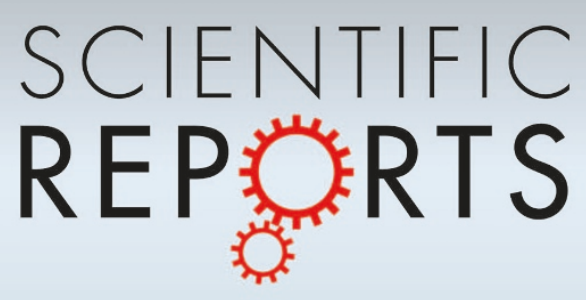

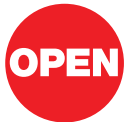

SUBJECT AREAS:

ATMOSPHERIC

DYNAMICS

CLIMATE-CHANGE IMPACTS

OCEAN SCIENCES

ENVIRONMENTAL SCIENCES

Received

19 September 2012

Accepted

13 February 2013

Published

22 March 2013

Correspondence and requests for materials should be addressed to S.Q.P. (speng@scsio. ac.cn)

\section{Does warmer China land attract more super typhoons?}

\author{
Xiangde $X u^{1,2}$, Shiqiu Peng ${ }^{2}$, Xiangjing Yang ${ }^{1,3}$, Hongxiong $X_{u^{1}}$, Daniel Q. Tong ${ }^{4}$, Dongxiao Wang $^{2}$, \\ Yudi Guo', Johnny C. L. Chan ${ }^{5}$, Lianshou Chen', Wei Yu', Yineng Li ${ }^{2}$, Zhijuan Lai ${ }^{2} \&$ Shengiun Zhang'
}

${ }^{1}$ State Key Laboratory of Severe Weather, Chinese Academy of Meteorological Sciences, Beijing 100081, China, ${ }^{2}$ State Key Laboratory of Tropical Oceanography, South China Sea Institute of Oceanology, Chinese Academy of Sciences, Guangzhou 510301, China, ${ }^{3}$ Chenzhou Meteorological Bureau of Hunan Province, Chenzhou 423000, China, ${ }^{4}$ Air Resources Laboratory, National Oceanic and Atmospheric Administration, Silver Springs, Maryland 20910, USA, ${ }^{5}$ School of Energy and Environment, City University of Hong Kong, Hong Kong, China.

Accurate prediction of where and when typhoons (or named hurricanes which form over the North Atlantic Ocean) will make landfall is critical to protecting human lives and properties. Although the traditional method of typhoon track prediction based on the steering flow theory has been proven to be an effective way in most situations, it slipped up in some cases. Our analysis of the long-term Chinese typhoon records reveals that typhoons, especially super typhoons (those with maximum sustained surface winds of greater than $\left.51 \mathrm{~ms}^{-1}\right)$, have a trend to make landfalls toward warmer land in China over the past 50 years (19602009). Numerical sensitivity experiments using an advanced atmospheric model further confirm this finding. Our finding suggests an alternative approach to predict the landfall tracks of the most devastating typhoons in the southeastern China.

(2) hina is one of the countries hit most frequently and severely by typhoons, strong tropical cyclones with maximum sustained surface winds of greater than $33 \mathrm{~ms}^{-1}$ that form over the Northwest Pacific Ocean. On average, China sees 7 to 8 landfalls of typhoons per year, impacting a population of 250 millions with property values over 10 trillion dollars ${ }^{1}$. With the rapid increase of economy and population in the southeastern coast of China, the loss of property and life in these regions caused by the landfalls of typhoons may increase in the future. During the past few decades, observational networks and numerical models have been used to predict typhoon landfall tracks ${ }^{2,3}$, but large uncertainties remain. One of the major uncertainties is the prediction of where the typhoon will make landfall, which is essential for local decision makers to choose among proper emergency measures, such as evacuation of coastal residents to avoid loss of life. In this paper we report a new indicator to the landfall locations of typhoons, in particular super typhoons that pose the most severe threat to human lives and properties.

Traditionally, typhoon tracks are predicted based on the steering flow theory, in which a typhoon is considered as an imbedded vortex in the height-averaged large-scale winds ${ }^{4-10}$. This scheme proves instrumental, producing reasonable forecasts of typhoon tracks in many cases. However, it has failed in several high profile cases ${ }^{11}$. In these cases, the landfalls of the super typhoons did not follow the steering flow, contradicting traditional wisdom. Other factors that control typhoon landfalls remain elusive.

Typhoons, as well other tropical cyclones, are conceived over warm ocean surfaces where plenty of energy and water vapor are available ${ }^{12}$. Typhoons are intensified when moving over warmer sea surface, while weakened over colder sea surface ${ }^{13-15}$. Compared to the widely recognized ocean surface effects, little is known of what role the land surface plays in regulating typhoon tracks. Specifically, it is unclear if warmer land surface can influence the tracks of typhoons and hence their landfalls. In this paper, we report our analysis of the relationship between land surface temperature (LST) and typhoon landfalls in China during the past 50 years (1960-2009). The long-term records of land surface temperature and typhoon tracks are retrieved from the quality assured archive collected and maintained by the Chinese Meteorology Administration (CMA).

\title{
Results
}

Fig. 1 shows the tracks of super typhoons and LST two days before landfalls in southeastern China for the decades of 1960, 1970s, 1980s, 1990s, and 2000s. It is interesting to see that, for 1960s, 1980s and 2000s, the majority of super typhoons clustered their tracks and make landfalls at a warmer area with LST higher than $31^{\circ} \mathrm{C}$, while the 

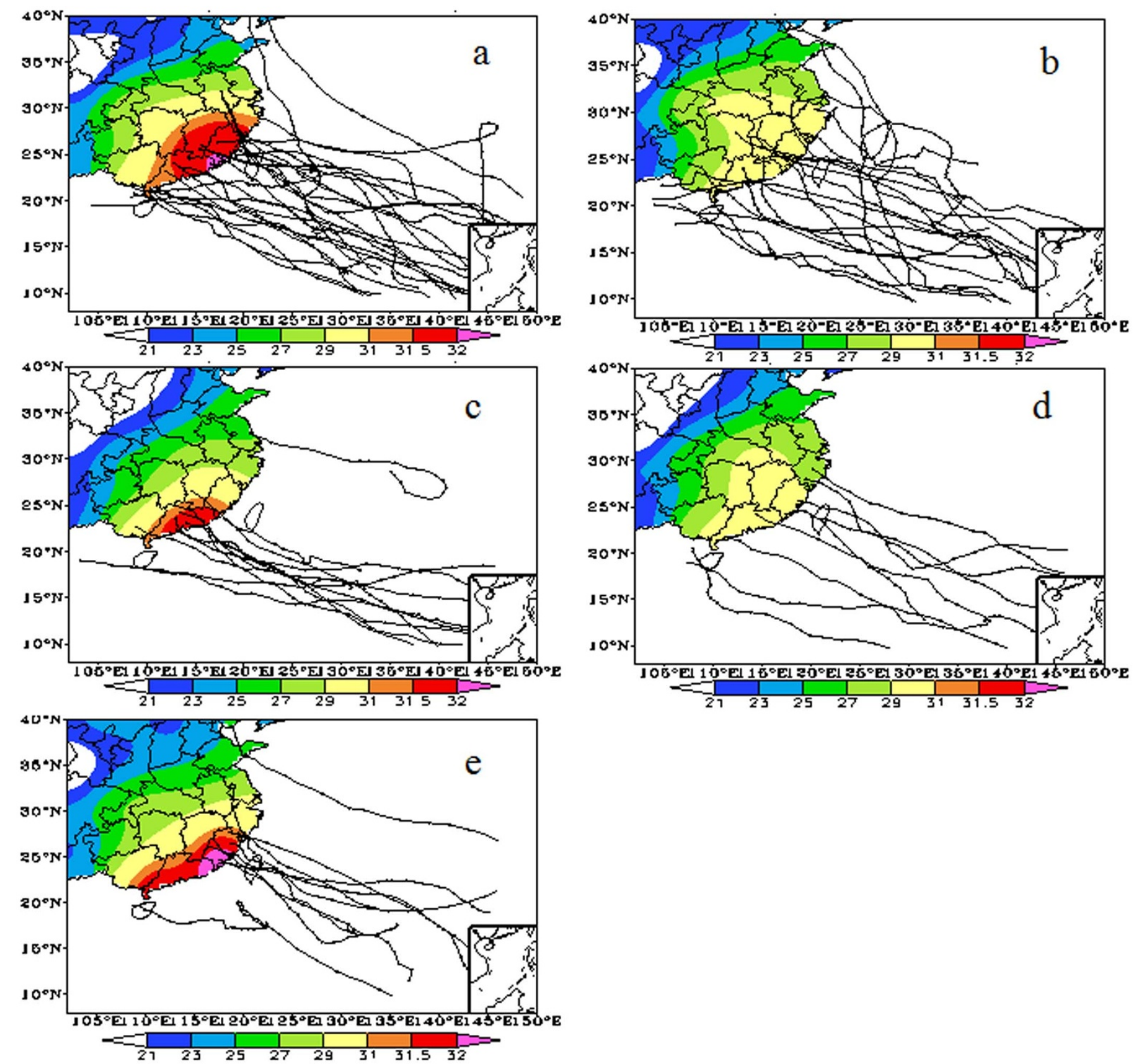

Figure 1 Tracks of super typhoons and composite land surface temperature (color shaded, unit: Celsius degree) two days before landfalls in the southeastern China for the 5 decades since 1960. (a) 1960s. (b) 1970s. (c) 1980s. (d) 1990s. (e) 2000s.

tracks of super typhoons show a diverse pattern for 1970s and 1990s when LST is lower than $31^{\circ} \mathrm{C}$. To illustrate more clearly the features of such a "warmward landfall", we examine the actual landfall tracks and the tracks predicted toward the highest land temperature points for all super typhoons during 1960-2009 (see Supplementary Fig. S2 online) and show four of them in Fig. 2 which have a recurvature feature before landfalls (see more cases in Supplementary Fig. S2 online). To make a comparison with the traditional track prediction method using steering flow theory, the direction of the corresponding steering flow is also shown in each panel. We define $\alpha_{1}$ as the track deviation angle for the track predicted toward the highest land temperature point and $\alpha_{2}$ as the one for the track predicted by the steering flow (see Supplementary Fig. S1 online). The point of the highest LST is determined within a coastal area of $30^{\circ} \times 30^{\circ}$ surrounding the cyclone center. For these super typhoons, the values of $\alpha_{1}$ are comparable to or even smaller than those of $\alpha_{2}$. The good agreement between the LST-predicted and actual landfall tracks suggests that, in addition to the environmental steering flow, the warmer land surface may be another important factor regulating the landfall tracks of tropical cyclones.

How significant is the land temperature indicator for typhoon landfalls? We further compare the LST-predicted tracks to the observed tracks for super typhoons as well as the weaker cyclones during 1960-2009. Those typhoons that only landed on Taiwan or Hainan Islands are excluded in this analysis, due to the lack of LST data for these islands. We bin all typhoons into three categories based on wind speed $|\vec{V}|:$ typhoons $(|\vec{V}|: 32.7 \sim 41.4 \mathrm{~m} / \mathrm{s})$, severe typhoons $(|\vec{V}|: 41.5 \sim 51 \mathrm{~m} / \mathrm{s})$, and super typhoons $(|\vec{V}|:>51 \mathrm{~m} / \mathrm{s})$. For each bin, these typhoons are further grouped based on their deviation angles, in which smaller deviation angle indicates better agreement between the LST-predicted tracks and the observed ones (see 

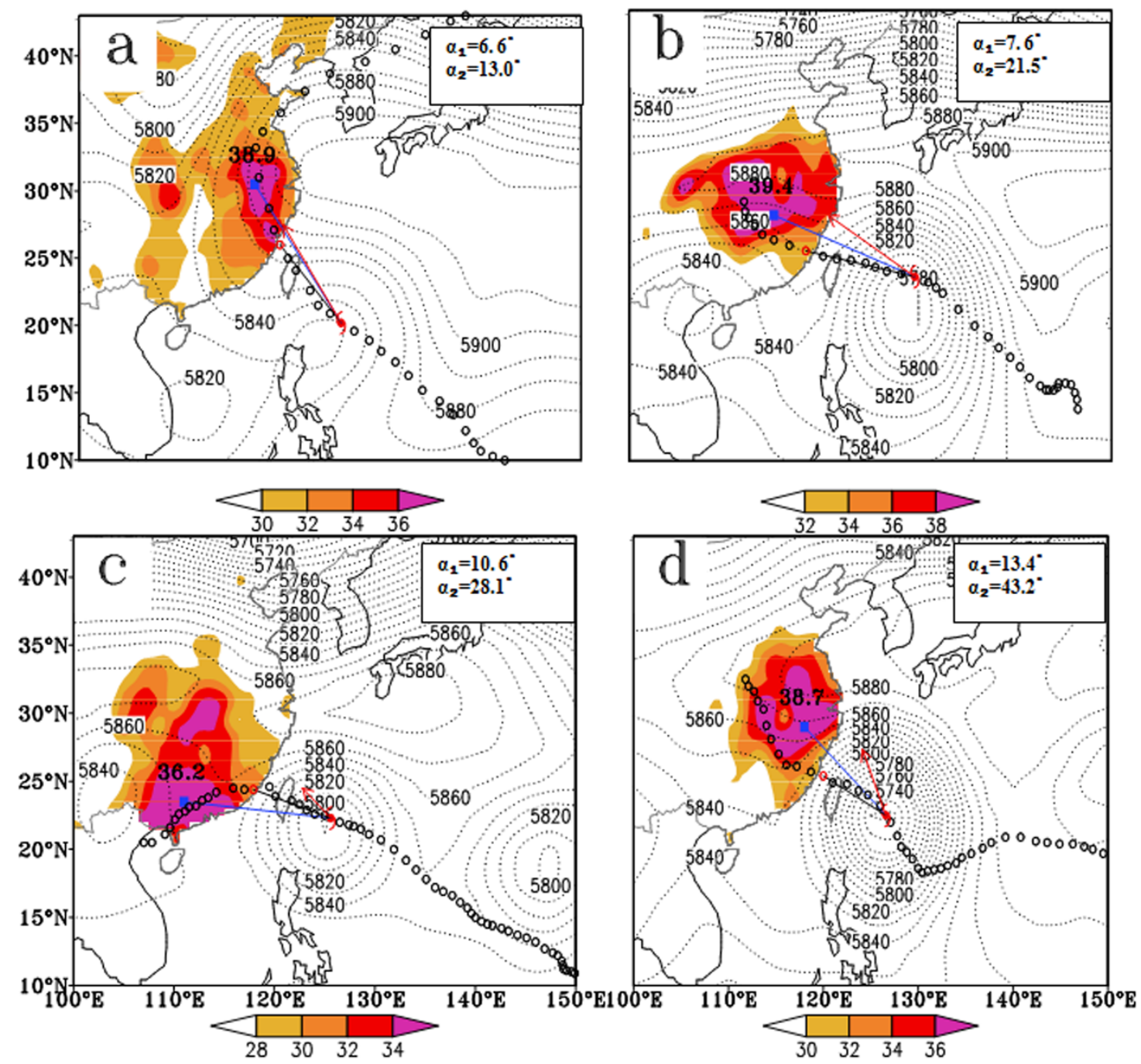

Figure $2 \mid$ The land surface temperature (color shaded, unit: Celsius degree), 500-hPa geopotential height (dashed contour, unit: gpm), the observed tracks, and the deviation angles of predicted tracks by the land surface temperature and the steering flow at the time of two days before landfalls of the super typhoons. (a) Opal (1962). (b) Billie (1976). (c) Omar (1992). (d) Herb (1996). A 9-point smoothing was applied to the land surface temperature for 3 times. The blue solid square denotes the location of highest land surface temperature within an area of $30^{\circ} \times 30^{\circ}$ surrounding the typhoon center at the time of two days before landfall, and the red empty dot denotes the location of landfall. The red arrow represents the steering flow. $\alpha_{1}$ and $\alpha_{2}$ are the deviation angles of the predicted tracks by the land surface temperature and the steering flow, respectively (see Supplementary Fig. S1 online).

Supplementary Table S1 online). Considering that the distance (L) between the typhoon position of two days before landing and the landing location may affect the calculated deviation angle, we further bin all typhoons into two categories based on the distance: $\mathrm{L}$
$<800 \mathrm{~km}$ and $\mathrm{L} \geq 800 \mathrm{~km}$. The statistical results are presented in Table 1. For $\mathrm{L}<800 \mathrm{~km}$, the percentages of all typhoons with deviation angle $\alpha_{1} \leq 15^{\circ}$ and $15^{\circ}<\alpha_{1} \leq 30^{\circ}$ account for $33 \%$ and $22 \%$, respectively; for $\mathrm{L}>800 \mathrm{~km}$, the corresponding percentages are $38 \%$

Table 1 | Statistics on the accuracy of the land-temperature-predicted (steering-flow-predicted) landfalls compared against the observed tracks for different categories of typhoons making landfalls on the mainland of China during 1960-2009. $\alpha_{1}\left(\right.$ or $\alpha_{2}$ ) is the deviation angle of the land-temperature-predicted (or the steering-flow-predicted) tracks compared against the observed tracks, and $L$ the distance between the typhoon position at the time of two days before landing and the landing location

\begin{tabular}{lccccc} 
& Category Of typhoons (No. of events) & $\alpha_{1}\left(\alpha_{2}\right) \leq 15^{\circ}$ & $15^{\circ}<\alpha_{1}\left(\alpha_{2}\right) \leq 30^{\circ}$ & $30^{\circ}<\alpha_{1}\left(\alpha_{2}\right) \leq 45^{\circ}$ & $45^{\circ}<\alpha_{1}\left(\alpha_{2}\right) \leq 90^{\circ}$ \\
\hline $\mathrm{L}<800 \mathrm{~km}$ & Typhoons (51) & $31 \%(33 \%)$ & $18 \%(28 \%)$ & $24 \%(18 \%)$ & $24 \%(20 \%)$ \\
& Severe typhoons (23) & $26 \%(17 \%)$ & $22 \%(35 \%)$ & $26 \%(26 \%)$ & $22 \%(17 \%)$ \\
& Super typhoon (30) & $40 \%(47 \%)$ & $27 \%(30 \%)$ & $20 \%(13 \%)$ & $13 \%(7 \%)$ \\
$\mathrm{L} \geq 800 \mathrm{~km}$ & Total (104) & $33 \%(33 \%)$ & $22 \%(31 \%)$ & $23 \%(19 \%)$ & $20 \%(15 \%)$ \\
& Typhoons (23) & $44 \%(35 \%)$ & $26 \%(39 \%)$ & $13 \%(17 \%)$ & $17 \%(9 \%)$ \\
& Severe typhoons (20) & $25 \%(30 \%)$ & $40 \%(55 \%)$ & $20 \%(15 \%)$ & $15 \%(0 \%)$ \\
& Super typhoons (29) & $45 \%(59 \%)$ & $31 \%(21 \%)$ & $7 \%(14 \%)$ & $17 \%(7 \%)$ \\
& Total (72) & $38 \%(41 \%)$ & $32 \%(38 \%)$ & $13 \%(15 \%)$ & $17 \%(5 \%)$ \\
\hline
\end{tabular}


Table 2 | The mean deviation angles $\bar{\alpha}_{1}\left(\bar{\alpha}_{2}\right)$ (unit: degree) of landtemperature-predicted (steering-flow-predicted) tracks compared against the observed tracks for different categories of typhoons making landfalls on the mainland of China during 1960-2009. These mean deviation angles $\bar{\alpha}_{1}\left(\bar{\alpha}_{2}\right)$ are averaged over all deviation angles $\alpha_{1}$ (or $\alpha_{2}$ ) from 4 days to 1 day before the landfalls of all typhoons located at a given range of latitudes

Category Of

typhoons

$$
10^{\circ}-15^{\circ}
$$$$
15^{\circ}-20^{\circ}
$$

$20^{\circ}-25$

$25^{\circ}-30^{\circ}$

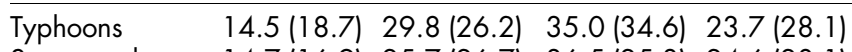

Severe typhoons 14.7 (16.9) 25.7 (26.7) 36.5 (25.3) $24.6(28.1)$

Super typhoons $11.2(14.9) \quad 18.4(19.6) \quad 23.0(18.5) \quad 39.5(29.6)$

Total

$13.8(17.3) \quad 25.3(24.3) \quad 30.8(26.1) \quad 30.0(28.7)$

and $32 \%$, respectively. For both $\mathrm{L}<800 \mathrm{~km}$ and $\mathrm{L} \geq 800 \mathrm{~km}$, super typhoons have the highest percentage for the smallest deviation angle $\left(\alpha_{1} \leq 15^{\circ}\right)$, indicating that super typhoons may move toward warmer land surface most likely. For comparison, the statistics for the deviation angle $\alpha_{2}$ of steering-flow-predicted tracks are also given in Table 1 (indicated in parentheses). It is surprising to find that the accuracy of the LST-predicted tracks is comparable to that of the steering-flow-predicted tracks (also see Supplementary Table S2 online). Table 2 shows the mean deviation angles of the predicted tracks by both the LST and the steering flow compared against the observed tracks for different categories of typhoons making landfalls at low-middle latitudes $\left(10^{\circ} \mathrm{N}-30^{\circ} \mathrm{N}\right)$. These mean deviation angles are obtained by making an average over all deviation angles $\left(\alpha_{1}\right.$ or $\left.\alpha_{2}\right)$ from 4 days to 1 day before the landfalls of all typhoons located at a given range of latitudes (with an interval of $5^{\circ}$ ) during 1960-2009. It is found that the mean value $\left(\bar{\alpha}_{1}\right)$ of $\alpha_{1}$ is comparable to that $\left(\bar{\alpha}_{2}\right)$ of $\alpha_{2}$ at each range of latitudes, and both $\bar{\alpha}_{1}$ and $\bar{\alpha}_{2}$ are smaller at low latitudes than in middle latitudes. For both the steering flow predictor and the LST predictor, the accuracy of track prediction for super typhoons appears to be higher than that for weaker ones except for the latitude range of $25^{\circ} \sim 30^{\circ}$. By examining the highest LST for super typhoons with $\alpha_{1}<15^{\circ}$ (with a total number of 25) during 1960-2009, it is found that there are 19 out of 25 with the LST higher than $31^{\circ} \mathrm{C}$, accounting for $76 \%$ of the total (see Supplementary Fig. S3 online). Therefore, based on Fig. 1 and Fig. S3 (as well as Fig. 5a), we may recommend the land surface temperature exceeding $31^{\circ} \mathrm{C}$ as a track predictor for super typhoons.

To investigate whether the LST predictor and the steering flow predictor are identical to each other, we calculate the percentages of the right (positive) or left (negative) deviation of predicted tracks by the two predictors for all typhoons during 1960-2009. The percentages are averaged from 4 days to 1 day before the landfall of each typhoon located at a given range of latitudes. The results are shown in Fig. 3. The LST-predicted tracks have a larger likelihood of deviating right at low latitudes than at middle latitudes while they tend to deviate left more likely at middle latitudes than at low latitudes. The situation for the tracks predicted by the steering flow is opposite to that for the tracks predicted by LST, i.e., they have a larger likelihood of deviating right at middle latitudes than at low latitudes while they tend to deviate left more likely at low latitudes than at middle latitudes. The reason may be primarily attributed to the meridional variation of the coriolis force, the so-called beta-effect, which is considered to have significant impacts on the movement of typhoons ${ }^{16-23}$. In the northern Hemisphere, barotropic modeling studies have shown that the beta-effect may make the cyclonic vortex move northwestward at a speed of $1-3 \mathrm{~ms}^{-1}$ due to the asymmetric coriolis force exerting on the cyclonic circulation and the inward convergent flows ${ }^{18-23}$. Because the relative position of a typhoon to the land surface is dependent on the latitude where it locates, the predicted moving direction of a typhoon at low latitudes using the LST predictor may be north-northwest most likely, while that at middle latitudes may be west-northwest most likely. Therefore, the beta-effect may make the actual tracks toward the west (left) at low latitudes and toward the north (right) at middle latitudes, i.e., the beta-effect may make the LST-predicted tracks deviate to the right at lower latitudes and to the left at higher latitudes more likely compared to their actual tracks. For the steering-flow predictor, the direction of the steering flow is also greatly dependent on the latitude: at low latitudes, the steering flow may mostly follow the westward flows at the southern edge of the subtropical high, thus the beta-effect may cause the actual tracks toward the north (right), i.e., make the steering-flow-predicted tracks deviate to the left more likely compared to their actual ones; at middle latitudes, the steering flow may mostly follow the northward flows at the western edge of the subtropical high, thus the beta-effect may cause the actual tracks toward the west (left), i.e., make the steering-flow-predicted tracks deviate to the right more likely compared to their actual ones. These results imply that, although the two predictors of the steering flow and the land surface temperature are not totally independent to each other, they are not identical to each other either, which makes the land surface temperature be a valuable predictor for typhoon tracks supplementary to the steering flow. The beta-effect is important and should be considered deliberately no matter using the LST predictor or the steering flow one.

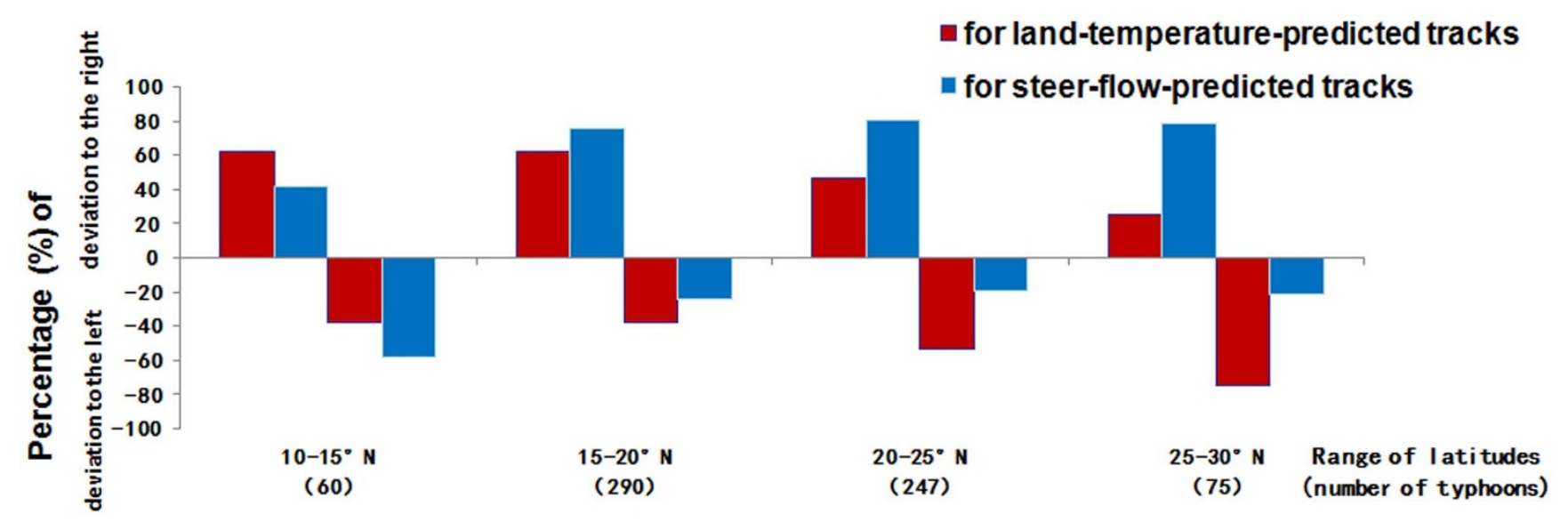

Figure 3 The percentages of the right (positive) or left (negative) deviation of predicted tracks by the land surface temperature and the steering flow compared against the observed tracks for all typhoons during 1960-1009. The percentages are averaged from 4 days to 1 day before the landfall of each typhoon located at different ranges of latitudes (with an interval of $5^{\circ}$ ). 

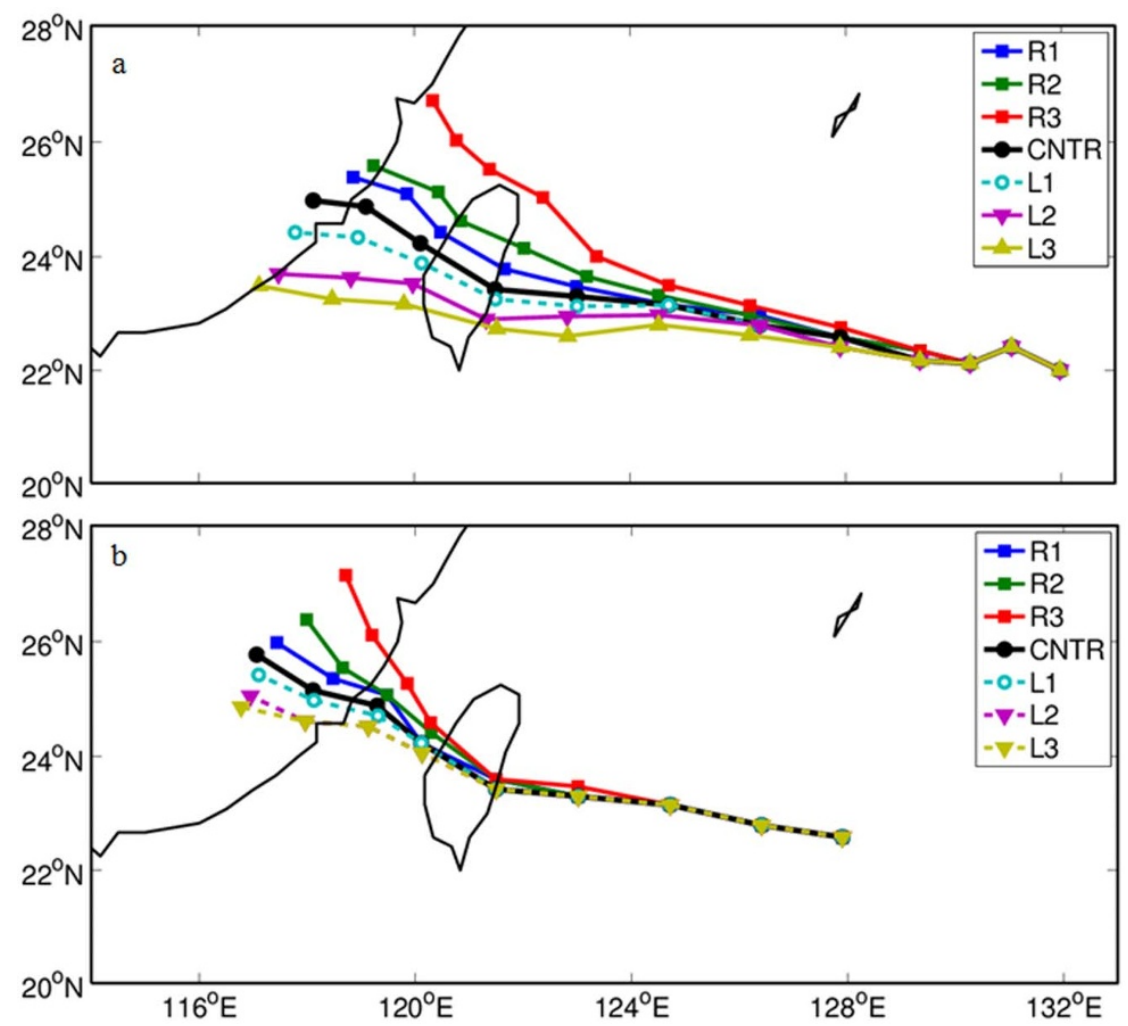

Figure $4 \mid$ The simulated tracks of Longwang (2005) from the control run (CNTR) and different sensitivity experiments R1, R2, R3, L1, L2, and L3. (a) Initializing at 0000 UTC 30 Sept. 2005 (3 days before landing). (b) Initializing at 0000 UTC 1 Oct. 2005 (2 days before landing). Here R1, R2, and R3 denote the experiments in which the land surface temperature is increased by $5 \mathrm{~K}, 10 \mathrm{~K}$ and $15 \mathrm{~K}$ at the initial time on the right hand side of the observed landing location, respectively, while L1, L2 and L3 denote the experiments in which the land surface temperature is increased by $5 \mathrm{~K}, 10 \mathrm{~K}$ and $15 \mathrm{~K}$ on the left hand side, respectively (see Supplementary Fig. S4 online for more details).

To further confirm our finding of the "warmward landing" trend of super typhoons, we conduct a set of numerical sensitivity experiments using an advanced atmospheric model called Weather and Research Forecasting (WRF) which includes comprehensive physical processes $^{24}$. We choose the super typhoon Longwang (2005) for our sensitivity experiments. We increase LST by $5 \mathrm{~K}, 10 \mathrm{~K}$ and $15 \mathrm{~K}$ on the right hand side (denoted as R1, R2 and R3) and the left hand side (denoted as L1, L2 and L3) of the observed landing location, respectively, at the time of 3 days and 2 days before the landfall of Landwang (2005), while keeping all the other circumstances the same as those of the control run (denoted as CNTR) (see Supplementary section 1.4 and Fig. S5 online). It is found that when increasing LST on the right (left), the typhoon track deviates to the right (left), and the more the LST is increased, the more the track deviates from CNTR (Fig. 4). The results of these numerical experiments confirm that super typhoons do have a trend to move toward the warmer land surface.

What is the mechanism of the "wardwarm landing" for typhoons? It is well known that tropical cyclones tend to move toward warmer sea surface to feed on moisture and energy ${ }^{12-15}$. We propose here that warmer land surface, with the intense moisture supplied by summertime Asian Monsoon ${ }^{25}$, plays a similar role in attracting typhoons to move toward warmer ground surface. Fig. 5a shows the variation of the averaged land surface temperature 10 days before and after the landfalls of typhoons and super typhoons with $\alpha_{1}<15^{\circ}$ during 1960 2009. For super typhoons with $\alpha_{1}<15^{\circ}$, it is found that LST starts to increase (up to $31^{\circ} \mathrm{C}$ ) as early as 4 days before landfall, but drops sharply near landfall and increases again after landfall. The drop of LST could be due to the latent heat release by the strong convection and the cooling by rainfall on the ground during the typhoon landfall. For typhoons, similar variation of LST is found but the increase of LST before landfall is relatively weaker than that for super typhoons, which indicates that the signal of LST as a track predictor for a typhoon is weaker than that for a super typhoon. One reason for this could be that the super typhoons, which contain tremendous moisture and energy, may have more intensified and farther-reaching cyclonic flows than weaker ones. These cyclonic flows could carry large amount of moisture to the warm land surface and thus favor the development of deep convection over the warm land (indicated by the large yellow arrows in Fig. 6). Therefore, the thermodynamical interactions between super typhoons and the warmer land surface could be more active and efficient, which makes the super typhoons move toward the warmer land surface more likely. Another reason could be that the vertical structure of the atmosphere over land is more unstable during super typhoons than that during typhoons, resulting in more deep convection over the warmer land surface. To verify this, we analyzed the temporal variation of the mean air temperature and water vapor anomalies during the landfalls of the super typhoons and typhoons with $\alpha_{1}<15^{\circ}$ (see Supplementary Fig. S7 online) as well as their differences (Fig. 5b) averaged over an area of $600 \mathrm{~km} \times 600 \mathrm{~km}$ centered at the warmest land surface two days before landfalls during 2000-2009. Relatively wetter and colder air is found over the land surface several days before the landfalls of super typhoons with $\alpha_{1}<15^{\circ}$, compared to those of typhoons with $\alpha_{1}<15^{\circ}$. Since the mean land surface temperature before the landfalls of super typhoons is generally higher than that of typhoons, larger soil-air temperature difference occurred with colder air over the warmer land surface (see Supplementary Fig. S19 online), which, accompanied with more moisture probably supplied by both the southwesterly East Asian Summer Monsoon (EASM) and the detrained flows at the outer edge of super typhoons, may increase the instability of the atmospheric planetary boundary layer (PBL) of the atmosphere. 

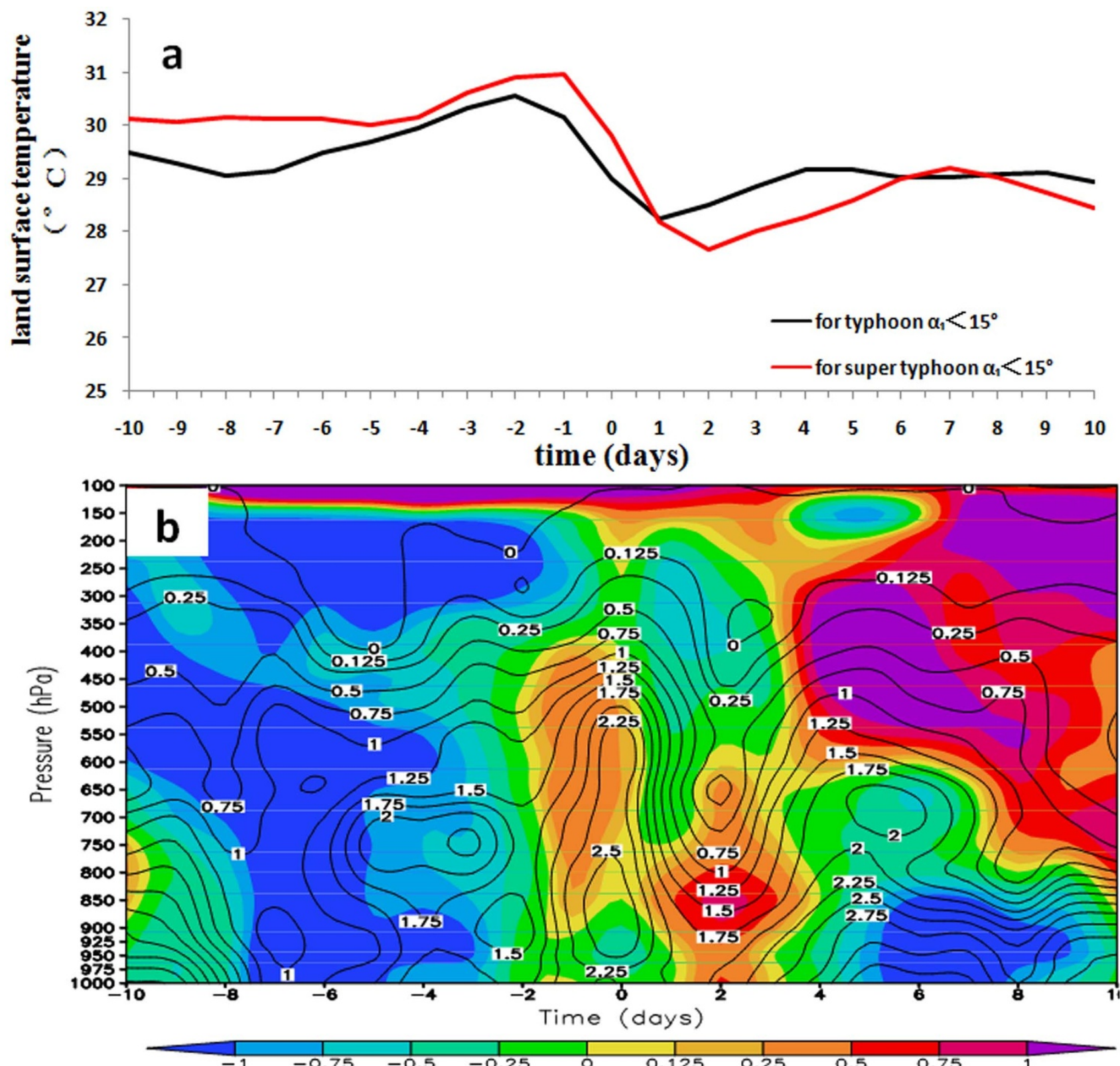

Figure 5 Temporal variations of mean land surface temperature, air temperature and moisture averaged over an area with radius of $600 \mathrm{~km}$ centered at the warmest land surface two days before landfall. (a) Mean land surface temperature (unit: Celsius degree) 10 days before and after the landfalls of typhoons (black curve) and super typhoons (red curve) with $\alpha_{1}<15^{\circ}$ during 1960-2009. (b) Time-height cross section of the differences of the air temperature (color shaded, unit: K) and moisture (contour, unit: $\mathrm{g} \cdot \mathrm{kg}^{-1}$ ) between the super typhoons and typhoons with $\alpha_{1}<15^{\circ}$ during $2000-2009$.

Such an unstable structure of the atmospheric PBL favors the occurrence of deep convection, which in turn feed the super typhoons with energy and moisture and thus "attract" the movement of the super typhoons. A diagnose of divergence and vorticity fields for the super typhoon Longwang (2005) using reanalysis data shows that the convergence center with positive vorticity of the super typhoon moved toward the convergence zone with positive vorticity over the warmer land surface and merged together (see Supplementary Figs. S8-S9 online). Analysis from the experimental results also indicates that, the increase of LST (sensitivity experiments L3 and R3) leads to a convergence of moisture flux and latent heat at the low-middle atmosphere and an increase of PBL height over the warmer land surface, which favors the development of deep convection and thus supplies enough moisture and energy for the super typhoon (see Supplementary Figs. S10-S12 online). We can see more clearly the thermal-dynamical interactions between a strong typhoon and the atmosphere over the warmer land surface by examining the temporal-spatial variations of the four critical meteorological parameters for several strong typhoons: the apparent heat source $\left(Q_{1}\right)$, apparent moisture sink $\left(Q_{2}\right)$, and the vertical motion (see Supplementary Figs. S13-S18 online). The high value centers of $Q_{1}, Q_{2}$ and vertical velocity of the super typhoons appear to move toward those over warmer land surface. We found their interactions can be best sketched with a conceptual model illustrated by Fig. 6 . During the typhoon season, the southwesterly EASM constantly transports water vapor from the southwestern sea to the warm land surface (indicated by the small white arrows) and the typhoon center (indicated by the large white arrows). There may be a convergence of moisture over the land where the moisture transported by EASM meets that transported by the detrained flows of the super typhoon (indicated by large yellow arrows). Meanwhile, warm land surface heats up the lower atmosphere, inducing stronger atmospheric convections. With the rise of 


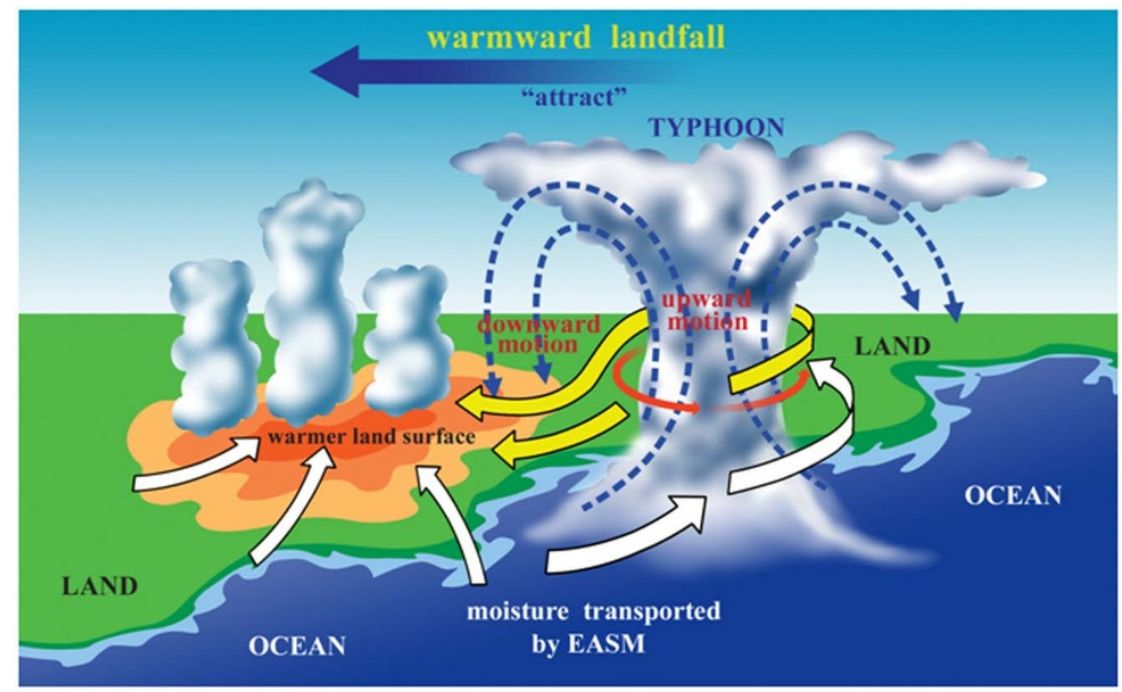

Figure 6 Cartoon of a conceptual model of the thermodynamic interactions between warmer land surface and a super typhoon approaching the coasts of China. The white and yellow arrows denote the moisture transportation by East Asian Summer Monsoon (EASM) and the detrained flows of the super typhoon, respectively.

warm and moist surface air into the middle or upper troposphere, a large amount of latent heat is released, forming a warm core with anomalous $Q_{1}$ and $Q_{2}$. To balance the upward motions, downward motions are also forced in the vicinity of the warm core. The intense heat and moisture over the warm land surface can feed typhoons in the same way as over the warm ocean surface, providing great incentives for typhoons to move toward the warmer land (see Supplementary Figs. S8-S18 online).

\section{Discussion}

It is worthy to note that the impact of warmer land surface on the super typhoon tracks is largely depended on the available moisture of the lower atmosphere that is necessary for the development of deep convection. In the southeastern China, the EASM brings plenty of water vapor from the ocean, which provides the necessary moisture for deep convection over the warmer land surface. In the regions where there is no moisture available, the impacts of warmer land surface on the super typhoon tracks may be small or negligible. Therefore, the applicability or accuracy of the LST predictor for typhoon tracks may be regionally dependent and a thorough investigation should be taken before using the LST as a TC track predictor for any other region.

Since land and air temperatures are intimately coupled, we also examined if air temperature can serve as a reliable indicator to typhoon landfall tracks. Among all cases investigated, we found no significant correlation between landfall locations and air temperature over these locations or their adjacent areas. Higher air temperature over land surface is often linked to lower relative humidity and less large-scale ascent or deep convection. The dry circulation anomalies inhibit tropical cyclones from approaching ${ }^{26}$. Further analysis indicates that there is a good relationship between landfall locations of super typhoons and soil-air temperature differences (see Supplementary Figs. S19-S21 online). The reason could be that, the large soil-air temperature differences, along with the abundant moisture supplied by the EASM, are conducive to the large-scale ascent and deep convection required for the development and maintenance of tropical cyclones.

It was estimated that half of the world's population will be living within $100 \mathrm{~km}$ of the coast by $2030^{27}$, which makes accurate prediction of the landfalls of super typhoons and hurricanes ever more important when making life-or-death decisions. It has been recognized that the mechanism to determine the movement of tropical cyclones is very complicated and a large part of it remains unresolved, especially for super typhoons. Although further investigation is still needed, our finding that super typhoons tend to make landfalls toward warmer land could be practically valuable to better cope with such potential natural disasters. Complementing the current steering flow theory, we propose the use of land surface temperature as a new indicator for improving landfall forecasts of super typhoons over southeastern China. Our results, while focusing on China, offer a new perspective on understanding the dynamic behaviors of the most powerful windstorms on Earth.

\section{Methods}

The land surface temperature used in this study is obtained from the CMA archive of daily-mean land surface temperature measured at 753 stations over China (which has denser distribution in coastal regions of China) and interpolated to regular grid points with a resolution of $0.5^{\circ} \times 0.5^{\circ}$ using the Cressman method ${ }^{28}$. A 9-point smoothing was applied 30 times to get the composite land surface temperature field at the time of 2 days before landfalls of each super typhoon in Fig. 1, and three times to get the individual land surface temperature field in Fig. 2. The result for each grid point after the 9-point smoothing is a weighted average of the grid point plus the 8 surrounding points with the weights of 1.0 (center point), 0.5 (the points at each side and above and below), and 0.3 (corner points). Any missing data points are not included in the sum; points beyond the grid boundary are considered to be missing. The location of highest land surface temperature 1-4 days before the landfall of a typhoon is identified by a searching algorithm within a coastal area of $30^{\circ} \times 30^{\circ}$ centered at typhoon center.

The reanalysis data from the National Centers for Environmental Prediction (NCEP) of the United States National Oceanic and Atmospheric Administration (US NOAA), available 4 times a day at 0000 UTC, 0600 UTC, 1200 UTC, and 1800 UTC with horizontal resolution of $2.5^{\circ} \times 2.5^{\circ}$ (before 2000) or $1^{\circ} \times 1^{\circ}$ (on/after 2000), are used to derive the daily-mean $500-\mathrm{hPa}$ potential height, the steering flow, the vertical velocity, the apparent heat source $\left(Q_{1}\right)$, the apparent moisture $\operatorname{sink}\left(Q_{2}\right)$, and the horizontal moisture flux $(\vec{F})$. The steering flow is obtained following the traditional method $^{8,29}$, which is a height- and area-averaged large-scale flow between $925 \mathrm{hPa}$ and $300 \mathrm{hPa}$ and over a ring-area with $3 \sim 8$ grids away from the typhoon center. $Q_{1}$, $Q_{2}$ and $\vec{F}$ are calculated using the following formulas:

$$
\begin{gathered}
Q_{1}=\frac{d S}{d t}=C_{p}\left[\frac{\partial T}{\partial t}+\overline{\vec{V}} \cdot \nabla T+\left(\frac{p}{p_{0}}\right)^{\frac{R}{c_{p}}} \cdot \overline{\bar{\omega}} \frac{\partial \bar{\theta}}{\partial p}\right]=Q_{11}+Q_{12}+Q_{13} . \\
Q_{2}=-L \frac{d q}{d t}=-L\left[\frac{\partial \bar{q}}{\partial t}+\nabla \cdot \overline{\bar{q}} \overrightarrow{\vec{V}}+\frac{\partial \overline{q \omega}}{\partial t}\right]=Q_{21}+Q_{22}+Q_{23} . \\
\vec{F}=\frac{1}{g} \vec{V} q .
\end{gathered}
$$

See the Supplementary Methods for detailed descriptions of each variable in the above formulas.

To see the sensitivity of the landfalling track of Longwang (2005) to the land surface temperature, we design a set of experiments by imposing different temperature increment $\Delta t$ on the right or left hand side of the observed landing location, and 
compare the predicted tracks with that by a control run without any change of land surface temperature. The following is a list of description for each experiment:

$\mathrm{R} 1: \Delta t=5 \mathrm{~K}$, on the right;

R2: $\Delta t=10 \mathrm{~K}$, on the right

R3: $\Delta t=15 \mathrm{~K}$, on the right;

L1: $\Delta t=5 \mathrm{~K}$, on the left;

L2: $\Delta t=10 \mathrm{~K}$, on the left

L3: $\Delta t=15 \mathrm{~K}$, on the left;

CNTR: control run, i.e., no change of land surface temperature.

Fig. S4 displays the distribution of land surface temperature after an increment $\Delta t=5 \mathrm{~K}$ is imposed on the right (R1) or left (L1) hand side of the observed landfalling location.

Two nested domains with horizontal resolutions of $60 \mathrm{~km}$ and $20 \mathrm{~km}$, respectively, are employed in all the experiments, with the central location of $\left(22.2^{\circ} \mathrm{N}, 124.0^{\circ} \mathrm{E}\right)$. There 36 layers in the vertical with the top at $10 \mathrm{hPa}$. Two sets of experiments, initializing at 0000 UTC 30 Sept. (about 3 days before landing) and at 0000 UTC 1 Oct. 2005 (about 2 days before landing) respectively, are performed. The initial conditions (IC) and boundary conditions (BC) for the first guess are generated from the NCEP Final Analysis dataset of the Global Forecast System with $1^{\circ} \times 1^{\circ}$ horizontal resolution and 6-h interval. See Supplementary Methods for detailed descriptions of the physical schemes employed in the experiments.

1. Liu, D. F. Pang, L. \& Xie, B. T. Typhoon disaster in China: prediction, prevention, and mitigation. Nat Hazards 49, 421-436 (2009).

2. Emanuel, K. Thermodynamic control of hurricane intensity. Nature 401, 665-669 (1999).

3. Saunders, M. A. \& Lea, A. S. Seasonal prediction of hurricane activity reaching the coast of the United States. Nature 434, 1005-1008 (2005)

4. Jordan, E. S. An observational study of the upper wind circulation around tropical storms. J. Meteor. 9, 340 (1952).

5. Miller, B. I. "The use of mean layer winds as a hurricane steering mechanism" (U.S. National Hurricane Research Project. Rep. No. 18, 1958).

6. George, J. E. \& Gray, W. M. Tropical cyclone motion and surrounding parameter relationships. J. Appl. Meteor. 15, 1252-1264 (1976).

7. Neumann, C. J. On the use of deep-layer-mean geopotential height fields in statistical prediction of tropical cyclone motion. 6th Conference on Hurricanes and Tropical Meteorology. Amer. Meteor. Soc. Boston, 32-38 (1979).

8. Chan, J. C. L. \& Gray, W. M. Tropical cyclone movement and surrounding flow relationships. Mon. Wea. Rev. 110, 1354-1374 (1982).

9. Holland, G. J. Tropical cyclone motion: A comparison of theory and observation. J. Atmos. Sci. 41, 68-75 (1984).

10. Dong, K. \& Newmann, C. J. The relationship between tropical cyclone motion and environmental geostrophic flows. Mon. Wea. Rev. 114, 115-122 (1986).

11. Wu, C.-C. Huang, T.-S. \& Chou, K.-H. Potential Vorticity Diagnosis of the Key Factors Affecting the Motion of Typhoon Sinlaku (2002). Mon. Wea. Rev. 132, 2084-2093 (2004).

12. Palmen, E. On the formation and structure of tropical cyclones. Geophyisca 3, 26-38 (1948).

13. Fisher, E. L. Hurricanes and the Sea-Surface Temperature Field. J. Atmos. Sci. 15 328-333 (1958).

14. Shapiro, L. J. \& Goldenberg, S. B. Atlantic sea surface temperatures and tropical cyclone formation. J. Clim. 11, 578-590 (1998).

15. Emanuel, K. An air-sea interaction theory for tropical cyclones. Part I: Steadystate maintenance. J. Atmos. Sci. 43, 585-604 (1986).

16. Rossby, C. G. On displacements and intensity changes of atmospheric vortices. J. Marine Res. 7, 157-187 (1948).

17. Rossby, C. G. On the mechanism for the release of potential energy in the atmosphere. J. Meteor. 6, 163-180 (1949).
18. Adem, J. A series solution for the barotropic vorticity equation and its application in the study of atmospheric vortices. Tellus 8, 364-372 (1956).

19. Anthes, R. A. \& Hoke, J. E. the effect of horizontal divergence and the latitudinal variation of the Coriolis Parameter on the drift of a model hurricane. Mon. Wea. Rev. 103, 757-763 (1975).

20. Holland, G. J. Tropical cyclone motion:Environmental interaction plus a Beta effect. J. Atmos. Sci. 40, 328-342 (1983)

21. Chan, J. C. L. \& Williams, R. T. Analytical and numerical studies of the beta-effect in the tropical cyclone motion. Part I: Zero mean flow. J. Atmos. Sci. 44, 1257-1265 (1987).

22. Fiorino, M. \& Elsberry, R. L. Some aspects of vortex structure in tropical cyclone motion. J. Atmos. Sci. 46, 979-990 (1989).

23. Carr, L. E. \& Elsberry, R. L. Models of Tropical Cyclone Wind Distribution and Beta-Effect Propagation for Application to Tropical Cyclone Track Forecasting. Mon. Wea. Rev. 125, 3190-3209 (1997).

24. Skamarock, W. C., Klemp, J. B., Dudhia, J., Gill, D. O., Barker, D. M., Duda, M. G., Huang, X.-Y., Wang, W. \& Powers, J. G. A description of the Advanced Research WRF version 3, NCAR Technical Note NCAR/TN-475 + STR. Boulder, Colorado (2008)

25. Zhou, X. X., Ding, Y. H. \& Wang, P. X. Moisture Transport in the Asian Summer Monsoon Region and Its Relationship with Summer Precipitation in China. Acto Meteorologica Sinica 66, 59-70 (2008).

26. Elsner, J. B. \& Jagger, T. H. Florida hurricane decline linked to surface warming 26th Conference on Hurricanes and Tropical Meteorology, 10A.6 (2004) (http://ams.confex.com/ams/26HURR/techprogram/paper_75095.htm).

27. Adger, W. N., Hughes, T. P., Folke, C., Carpenter, S. T. \& Rockstrom, J. Socialecological resilience to coastal disasters. Science 309, 1036-1039 (2005).

28. Cressman, G. An operational objective analysis system. Mon. Wea. Rev. 87, 367-374 (1959)

29. Evans, J. L., Holland, G. J. \& Elsberry, R. L. Interactions between a barotropic vortex and an idealized subtropical ridge. Part I: Vortex Motion. J. Atmos. Sci. 48 301-314 (1991)

\section{Acknowledgements}

This work was jointly supported by the MOST of China (973 Projects with Grant No. 2009CB421504 \& 2011CB403505), the Innovation Key Program of the Chinese Academy of Sciences (Grant No. KZCX2-EW-208), National Natural Science Foundation of China (Grant No. 41076009 \& 41075037), and the Hundred Talent Program of the Chinese Academy of Sciences. The Grid Analysis and Display System (GrADS) was employed to plot most of the figures.

\section{Author contributions}

X. X. and S.P. designed the study and analyzed the results. X.Y. and W.Y. collected the data and did the statistical calculation. H.X. performed the numerical experiments. S.P. and D.Q.T. wrote the manuscript. D.W., J.C., L.C. and L.C. gave some conceptual advice. Y.G., Y.L., Z.L. and S.Z. provided technical support for programming and figure-plotting.

\section{Additional information}

Supplementary information accompanies this paper at http://www.nature.com/ scientificreports

Competing financial interests: The authors declare no competing financial interests. License: This work is licensed under a Creative Commons

Attribution-NonCommercial-NoDerivs 3.0 Unported License. To view a copy of this license, visit http://creativecommons.org/licenses/by-nc-nd/3.0/

How to cite this article: $\mathrm{Xu}, \mathrm{X}$. et al. Does warmer China land attract more super typhoons? Sci. Rep. 3, 1522; DOI:10.1038/srep01522 (2013). 